\title{
Loss of laminin and of the laminin receptor integrin subunit $\alpha 6$ in situ correlates with cytokine induced down regulation of $\alpha 6$ on fibroblast-like synoviocytes from rheumatoid arthritis
}

\author{
N Rinaldi, T F E Barth, D Weis, M Schwarz-Eywill, A Pezzutto, M Lukoschek, D Brocai, \\ B Brado
}

\begin{abstract}
Objective-To investigate in situ the expression of the integrin receptor subunits $\alpha 6$ and $\beta 1$ and the distribution of the ligand laminin in the synovia from osteoarthritis (OA) and rheumatoid arthritis (RA) patients and to study the effect of cytokines and antirheumatic drugs on the expression of the $\alpha 6$ and $\beta 1$ integrin subunits on long term cultures of fibroblast-like synoviocytes (FBS) derived from OA and RA. Methods-The expression of the $\alpha 6$ and $\beta 1$ integrin subunits and the distribution of laminin were examined immunohistochemically in normal synovia and in synovia from patients with $O A$ and RA. The effect of proinflammatory cytokines (IL1 $\beta$ and $T N F(\alpha)$, and of antirheumatic drugs (salicylic acid, dexamethasone, and methotrexate) on the $\alpha 6$ and $\beta 1$ expression of cultured normal FBS and FBS from patients with $O A$ and RA was determined by flow cytometry.
\end{abstract}

Results-In normal synovia and in OA synovia samples with a low grade of inflammation, synovial lining cells (SLC) showed a parallel expression and distribution of $\alpha 6$ and laminin. In synovia samples of $\mathrm{OA}$ with a higher grade of inflammation and in the majority of RA synovia samples laminin was pericellularly distributed in a low number of SLC, whereas $\alpha 6$ was expressed on the surface of a high number of SLC. In RA synovia samples with severe inflammatory changes the gradual loss of laminin generally corresponded to a decrease of the $\alpha 6$ integrin subunit. $\beta 1$ was always strongly expressed in all synovia samples detected. Proinflammatory cytokines up regulated the expression of $\alpha 6$ and $\beta 1$ on OA-FBS, whereas these effectors decreased $\alpha 6$ and $\beta 1$ on RA-FBS. In contrast, antirheumatic drugs, in particular methotrexate and dexamethasone, reduced the expression of $\alpha 6$ and $\beta 1$ on OA-FBS, whereas the same treatment on RA-FBS stimulated the expression of these integrin subunits.

Conclusion-The gradual loss of laminin in chronic synovitis may contribute to the altered expression of $\alpha 6$ in SLC. IL1 $\beta$ and TNF $\alpha$ down regulated the expression of the $\alpha 6$ and $\beta 1$ integrin subunits on long term cultures of FBS derived from RA. Therefore, these cytokines may be among the effectors regulating the expression of the $\alpha 6$ integrin subunit in SLC in vivo. As antirheumatic drugs increase the expression of $\alpha 6$ on RA-FBS, the presence of the laminin receptor may confer a protective effect on the synovia in vivo.

(Ann Rheum Dis 1998;57:559-565)

Normal synovia is organised into an intimal layer of synovial lining cells and a subintima composed of fat and connective tissue that contains blood vessels and scattered leucocytes. ${ }^{1}$ Although, ultrastructurally no distinct basement membrane separates synovial lining cells (SLC) from the subintima, it has been shown immunhistochemically that different extracellular matrix proteins like laminin, entactin, and collagen type IV present a defined distribution in the synovium. ${ }^{2}$ This network of extracellular matrix components might provide an anchoring basement membrane for the synovia. The structural organisation of the normal synovia is stabilised through cell-cell and cell-matrix interactions mediated, in part, via a large family of cell surface adhesion molecules known as integrins. $^{3}{ }^{4}$ Integrins consist of an $\alpha / \beta$ heterodimer. Through the extracellular receptor domain, which is built up by both subunits, and the cytoplasmic portion of the $\beta$ subunit, which interacts with talin and $\alpha$ actin, integrins connect the extracellular milieu with the cytoskeleton. ${ }^{5}$ In chronic synovitis inflammatory changes contribute to the remodelling process of the extracellular matrix. ${ }^{67}$ These changes in the composition of the extracellular matrix might lead to an alteration of the cell-matrix interaction and to an altered regulation of the integrin expression of the cells.

The aim of this study was to determine immunohistologically the distribution of the basement membrane component laminin and of the corresponding adhesion receptor $\alpha 6 \beta 1$ in chronic synovitis in situ. We observed changes associated with the intensity and the aetiology of inflammation. As cytokines have been recognised as important factors involved in the regulation of integrin expression, we investigated the effect of various cytokines and of antirheumatic drugs on the expression of $\alpha 6$ and $\beta 1$ on fibroblast-like synoviocytes in vitro by flow cytometry. The differential effect of cytokines on the expression of $\alpha 6$ and $\beta 1$ was related to the aetiology of the arthritic process.
Accepted for publication 8 July 1998 
Table 1 Inflammation score, clinical and immunostaining data in synovial lining cells of normal synovia, of $O A$ synovia and of $R A$ synovia

\begin{tabular}{|c|c|c|c|c|c|c|c|c|c|}
\hline & $E S R$ & $R F$ & $\begin{array}{l}\text { Antirheumatic } \\
\text { treatment }\end{array}$ & $\begin{array}{l}\text { Site of } \\
\text { synovia }\end{array}$ & $\begin{array}{l}\text { Inflammation } \\
\text { score }\end{array}$ & $\begin{array}{l}\text { Synoviocyte } \\
\text { hyperplasia }\end{array}$ & Laminin & $a 6$ & $\beta 1$ \\
\hline \multicolumn{10}{|c|}{ Normal synovia } \\
\hline Sample 1 & - & & - & knee & 3 & 1 & $(4+)$ & $4+$ & $4+$ \\
\hline Sample 2 & - & & - & knee & 3 & 1 & $(4+)$ & $4+$ & $4+$ \\
\hline \multicolumn{10}{|l|}{ OA synovia } \\
\hline Sample 3 & 7 & & no & knee & 5 & 2 & $(3+)$ & $4+$ & $4+$ \\
\hline Sample 4 & 2 & & no & knee & 8 & 2 & $(1+)$ & $1+$ & $4+$ \\
\hline Sample 5 & 20 & & no & hip & 7 & 1 & $(4+)$ & $4+$ & $4+$ \\
\hline Sample 6 & 2 & & no & hip & 11 & 1 & $(3+)$ & $3+$ & $4+$ \\
\hline Sample 7 & 30 & & no & hip & 4 & 1 & $(2+)$ & $3+$ & $4+$ \\
\hline Sample 8 & 3 & & no & hip & 4 & 1 & $(3+)$ & $(3+)$ & $4+$ \\
\hline Sample 9 & 20 & & no & hip & 6 & 1 & $(3+)$ & $4+$ & $4+$ \\
\hline Sample 10 & ND & & no & knee & 4 & 1 & $(2+)$ & $4+$ & $4+$ \\
\hline Sample 11 & 20 & & no & knee & 7 & 1 & $(1+)$ & $3+$ & $4+$ \\
\hline Sample 12 & 6 & & NSAID & hip & 8 & 1 & $(1+)$ & $(1+)$ & $4+$ \\
\hline Sample 13 & 5 & & no & hip & 5 & 1 & $(1+)$ & $1+$ & $(3+)$ \\
\hline Sample 14 & 2 & & NSAID & hip & 7 & 1 & $(4+)$ & $4+$ & $4+$ \\
\hline Sample 15 & 18 & & no & hip & 6 & 2 & $(1+)$ & $(3+)$ & $4+$ \\
\hline Sample 16 & 3 & & NSAID & hip & 4 & 1 & $(3+)$ & $4+$ & $(3+)$ \\
\hline Sample 17 & ND & & no & hip & 7 & 2 & $(1+)$ & $(1+)$ & $(2+)$ \\
\hline \multicolumn{10}{|l|}{ RA synovia } \\
\hline Sample 18 & 18 & + & gold & knee & 7 & 1 & $(2+)$ & $4+$ & $4+$ \\
\hline Sample 19 & 22 & + & gold & wrist & 11 & 1 & $(2+)$ & $4+$ & $3+$ \\
\hline Sample 20 & 9 & ND & methotrexate & wrist & 15 & 4 & - & $(2+)$ & $4+$ \\
\hline Sample 21 & 20 & + & methotrexate & wrist & 9 & 2 & $3+$ & $4+$ & $4+$ \\
\hline Sample 22 & 35 & - & methotrexate & wrist & 9 & 1 & $(3+)$ & $3+$ & $3+$ \\
\hline Sample 23 & 25. & + & gold & knee & 11 & 1 & $(1+)$ & $2+$ & $4+$ \\
\hline Sample 24 & 16 & + & NSAID & wrist & 12 & 3 & - & - & $4+$ \\
\hline Sample 25 & 44 & + & methotrexate & knee & 9 & 1 & $(1+)$ & $(1+)$ & $4+$ \\
\hline Sample 26 & 40 & ND & methotrexate & hip & 8 & 2 & $(2+)$ & $(3+)$ & $4+$ \\
\hline Sample 27 & 34 & + & gold & knee & 13 & 2 & $(1+)$ & $2+$ & $(2+)$ \\
\hline Sample 28 & 4 & - & methotrexate & hip & 4 & 3 & $(2+)$ & $(2+)$ & $4+$ \\
\hline Sample 29 & 54 & + & methotrexate & knee & 6 & 1 & $(2+)$ & $(3+)$ & $(3+)$ \\
\hline Sample 30 & 21 & - & methotrexate & elbow & 15 & 3 & $(1+)$ & $3+$ & $4+$ \\
\hline Sample 31 & 17 & ND & methotrexate & knee & 10 & 1 & $(1+)$ & $2+$ & $4+$ \\
\hline Sample 32 & 33 & - & NSAID & wrist & 12 & 2 & $(2+)$ & $4+$ & $4+$ \\
\hline Sample 33 & 80 & + & penicillamine & knee & 15 & 4 & - & $(1+)$ & $4+$ \\
\hline Sample 34 & 20 & ND & gold & knee & 15 & 4 & - & $(1+)$ & $4+$ \\
\hline Sample 35 & 14 & + & methotrexate & hip & 10 & 2 & $(1+)$ & $1+$ & $4+$ \\
\hline Sample 36 & 20 & ND & penicillamine & knee & 8 & 1 & $(3+)$ & $3+$ & $4+$ \\
\hline
\end{tabular}

Scoring of cell reaction: $4+, 75-100 \%$ cells positive, $3+, 50-75 \%$ cells positive, $2+, 25-50 \%$ cells positive, $1+, 1-25 \%$ cells positive, ( ), low level of antigen expression,-, all cells negative. Scoring of synovial lining layers: 1, 1-3 cell layers, 2, 4-5 cell layers, 3, 6-8 cell layers, $4, \geqslant 9$ cell layers.

ESR, erythrocyte sedimentation rate; RF, rheumatoid factor; NSAID, non-steroidal anti-inflammatory drug; ND, not determined.

Methods

SYNOVIAL TISSUE

Synovial tissues were obtained at arthroplasty from different joints (table 1 ) of 23 patients with rheumatoid arthritis (RA) and 18 patients with osteoarthritis (OA) (according to the criteria of the American College of Rheumatology, formerly, the American Association of Rheumatology). ${ }^{89}$ Laboratory tests, erythrocyte sedimentation rate, and rheumatoid factor are reported in table 1 . Normal specimens were obtained at necropsy from two patients without arthritis who died of unrelated causes. Necropsy specimens were taken within six hours of death. Nineteen specimens of patients with RA and 15 synovia specimens of patients with $\mathrm{OA}$ were quick frozen in liquid nitrogen and stored at $-70^{\circ} \mathrm{C}$, whereas the other specimens (from three patients with OA and from four patients with RA) were processed immediately after excision and prepared for fibroblastlike synoviocytes (FBS) culture. A synovial fluid sample was collected from a patient with RA into tubes containing EDTA (Biochrom, Berlin, Germany) and stored at $-70^{\circ} \mathrm{C}$.

FBS ISOLATION

In vitro cultures of FBS were obtained by standard methods, as previously described. ${ }^{10}$ The tissue was minced into small pieces and digested with collagenase type Ia (Sigma Chemical Company, St Louis, MO, USA) in serum free basal Iscove medium (SeromedBiochrom, Berlin, Germany). The samples were washed and suspended in basal Iscove medium, supplemented with $10 \%$ fetal calf serum (FCS, Bio Pro, Karlsruhe, Germany) and with penicillin-streptomycin-amphotericin B (10 units $/ \mathrm{ml}, 10 \mathrm{mg} / \mathrm{ml}$ and $0.25 \mathrm{mg} / \mathrm{ml}$ ). Finally, isolated cells were cultured in a humidified $5 \%$ carbon dioxide atmosphere. After overnight culture, non-adherent cells were removed. Fresh medium was added and the incubation continued. At confluence, cells were trypsinised, split at 1:3 ratio, and recultured. The medium was changed twice each week. FBS from passages 3 to 9 were used in these experiments. Each of the three cultures of OA-FBS and the four cultures of RA-FBS were detached with EDTA $(0.02 \%)$ and centrifuged at $1000 \mathrm{rpm}$ for five minutes. The cultured cells comprised a homogeneous population of FBS with respect to morphological and immunocytochemical criteria $(<1 \%$ $\mathrm{CD} 11 \mathrm{~b}+,<1 \% \mathrm{CD} 11 \mathrm{a}+,<1 \% \mathrm{CD} 11 \mathrm{c}+,<$ $1 \%$ CD $53+,<1 \%$ CD $3+,<1 \%$ Factor VIII+).

STIMULATION OF FBS

FBS cultures from three patients with OA and from four patients with RA were cultured for 72 hours in the presence of cytokines and/or of antirheumatic drugs. One FBS culture (OA-FBS 1) was stimulated simultaneously with cytokines and drugs. Pilot studies were 
performed to test the concentration of cytokines and drugs showing the most relevant effect on the expression of $\alpha 6$ and $\beta 1$. On the basis of these data we used the following concentrations: IL1 $\beta$ (Genzyme; Cambridge, $\mathrm{MA}$ ): $40 \mathrm{U} / \mathrm{ml}$; $\mathrm{TNF} \alpha$ (Knoll; Ludwigshafen, Germany): $10 \mathrm{ng} / \mathrm{ml}$, salicylic acid (Sigma): $200 \mu \mathrm{g} / \mathrm{ml}$; dexamethasone (Sigma): $500 \mathrm{nM}$; methotrexate (Sigma): $100 \mu \mathrm{M}$. In four experiments FBS cultures were cultured for 72 hours in the presence of basal Iscove's medium, supplemented with $10 \%$ supernatant of synovial fluid from a patient with RA. At the end of the incubation, cells were washed with PBS, and detached with EDTA. Cell viability was measured by the trypan blue exclusion test that was $>95 \%$. Before analysis, cells were washed twice in PBS and resuspended in RPMI 1640 (Gibco, Paisley, Scotland, UK).

REAGENTS

The monoclonal primary antibody (mAb) $\mathrm{GOH} 3$ (anti- $\alpha 6$; IgG2a) and the anti-laminin $\mathrm{mAb} 4 \mathrm{C} 12$ (IgG1) were purchased from Dianova-Immunotech (Hamburg, Germany). The mAb P4C10 (anti- $\beta 1$; IgG1) was obtained from Telios (San Diego, USA). A polyclonal biotinylated sheep antibody to mouse Ig (reactive with all mouse and rat isotypes), a polyclonal biotinylated sheep antibody to rat Ig for detection of rat derived $\mathrm{mAb} \mathrm{GOH} 3$ and 4C12 and a streptavidin-biotinylated peroxidase complex were purchased from Amersham (High Wycombe, UK). 3-amino-9-ethylcarbazole (AEC) and N'N-dimethylformamide (DMF) were obtained from Sigma. An anticytomegalovirus antibody (clone $\mathrm{CCH} 2 ; \mathrm{IgG} 1$ ) was used as a negative control and was obtained from Dako (Glostrup, Denmark).

IMMUNOSTAINING PROCEDURE

Serial frozen sections of about $1 \mathrm{~cm}^{2}$ in area and 4-6 $\mu \mathrm{m}$ in thickness were air dried overnight, fixed in acetone for 10 minutes at room temperature, and immunostained immediately or after storage at $-20^{\circ} \mathrm{C}$ for $1-3$ weeks. Immunostaining procedures were carried out according to standard methods as previously described. ${ }^{11}$ Briefly, for immunohistochemistry after rehydration with phosphate buffered saline solution (PBS; $\mathrm{pH}$ 7.5), the frozen sections were incubated for one hour with primary mAbs. The primary $\mathrm{mAbs}$ were used in a protein concentration of about $5 \mu \mathrm{g} / \mathrm{ml}$ PBS. The sections were then incubated with biotinylated antimouse or antirat antibody $(20 \mu \mathrm{g} / \mathrm{ml}$ protein $)$ and streptavidin/biotin-peroxidase complex (10 $\mu \mathrm{g} / \mathrm{ml}$ protein) for 30 minutes, respectively. All incubation steps were carried out in a humid chamber at room temperature. Between each incubation step the sections were rinsed twice in PBS. Using AEC as the chromogen $(0.4 \mathrm{mg} / \mathrm{ml}$ in $0.1 \mathrm{~mol} / 1$ acetate buffer, $\mathrm{pH} 5,0$, with $5 \%$ $\mathrm{DMF}$ and $0.01 \%$ hydrogen peroxide for about 20 minutes), the peroxidase reaction caused an intense red precipitate. The sections were then rinsed in tap water, counterstained with Harris' haematoxylin, and mounted with glycerol gelatin. Each series of frozen sections contained a negative control without the primary reagent and the control anti-cytomegalovirus antibody. No staining was observed in the control slides except for scattered granulocytes whose endogenous peroxidase was not blocked to permit optimal antigenicity. This reactivity was disregarded during evaluation. In tissue sections, strongly stained endothelial cells, or lymphocytes, or stromal cells, or all three, always present in combinations characteristic of the respective antigen under study, served as intrinsic positive controls.

CONTROLS AND EVALUATION OF ANTIGEN EXPRESSION

SLC staining in tissue sections was evaluated in a semiquantitative manner: + , indicates strong intensity of the detected antigen; $(+)$, is regarded as weak intensity of antigen expression. The absence of antigen was symbolised as -. Whenever the staining intensity within FBS was heterogeneous, a simple semi-quantitative statement was made: $4+$, meaning $75-100 \%$ cells positive; $3+$, meaning $50-75 \%$ cells positive; $2+$, meaning $25-50 \%$ cells positive; $1+$, meaning $1-25 \%$ cells positive.

DEGREE OF INFLAMMATION

Haematoxylin and eosin stained sections from each synovial specimen were scored for the degree of inflammation by two independent observers, according to Rooney et al. ${ }^{12}$ Synovial lining cell depth was determined, and the results were recorded as 1 (1-3 cell layers), 2 ( $4-5$ cell layers), 3 ( $6-8$ cell layers), and 4 ( $\geqslant 9$ cell layers). Proliferating blood vessels were determined by immunostaining using anti-von Willebrand Factor (Dako, Glostrup, Denmark) and recorded as $1+,(0-5$ vessels/high power field $(\mathrm{HPF})(100 \times)) 2+(5-10$ vessels/HPF $)$, $3+(10-20$ vessels/HPF), and $4+(>20$ vessels/ $\mathrm{HPF})$. The size of lymphoid aggregates on stained sections was recorded as $0+$ (no aggregates), $1+(1-20$ cells/HPF $)$, and $2+(>20$ cells/HPF). The number of vessels showing perivascular infiltrates was determined for the final score and regarded as $1+(1-25 \%$ of the vessels involved), $2+(25-50 \%$ of the vessels involved), $3+(50-75 \%$ of the vessels involved), and $4+(75-100 \%$ of the vessels involved). The degree of fibrosis and the presence of necrosis were assessed. Sections containing $<10 \%$ fibrous tissue in the membrane sublining layers were considered normal and graded 1 . Sections containing $\geqslant 10-50 \%$ fibrous tissue were scored as $2+$, whereas sections with $\geqslant 50 \%$ fibrous tissue were graded as $3+$. The absence of necrosis was scored 0 . The presence of necrosis was scored $2+$. The scores of the individual items were added, and the sum was used as an estimate of the overall intensity of inflammation using histological criteria (range of possible values 3-19).

\section{FLOW CYTOMETRIC ANALYSIS}

FBS $\left(1 \times 10^{6}\right)$ were suspended in RPMI 1640, $10 \%$ FCS, $0.1 \%$ NaH3 (Merck, Darmstadt, Germany), and 2\% HEPES buffer (SeromedBiochrom). The expression of $\alpha 6$ and $\beta 1$ was assayed by flow cytometry, performed on a FACScan (Becton Dickinson) using the Lysis 

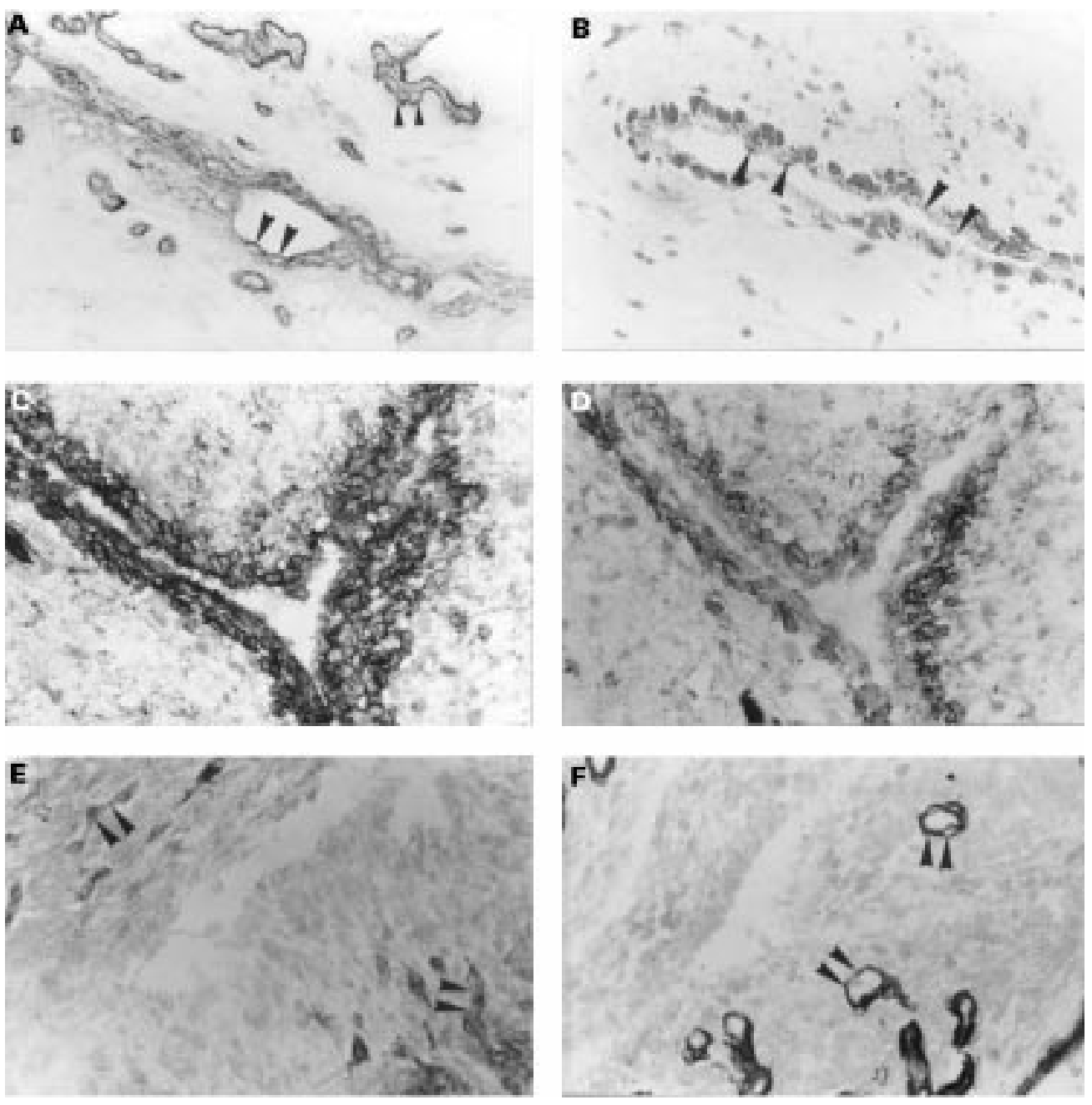

Figure 1 Expression of a6 integrin subunits and detection of laminin in SLC of normal synovia ( $A$ and B), in SLC of $O A$ synovia $(C$ and $D)$, and in SLC of RA synovia ( $E$ and $F)$. Normal synovia (sample 1$):(A)$ Normal, non-inflamed $S L C$ are strongly positive for the a6 integrin subunit (arrowheads). Endothelial cells of small vessels are also a6 positive (small arrowheads). (B) Normal SLC are organised in a monolayer and show a weak staining of laminin (arrowheads). $O A$ (serial sections, sample 10): (C) SLC form a multicellular laver and express the $a 6$ subunit in all cells. (D) Laminin is detected in about 50\% of SLC predominantly in the basal portion of the synovial layer. RA (sample 26): (E) hyperplastic $S L C$ are a6 negative. A few stromal cells and endothelial cells (arrowheads) are a6 positive serving as a positive control of the immune reaction. (F) Laminin is negative in the hyperplastic synovial layer of a rheumatoid synovia. Small vessels are strongly laminin positive (arrowheads); original magnification $\times 150$.

II Software. W6/32, a monoclonal antibody that reacts with the HLA-A,B,C/ $\beta 2 \mathrm{~m}-$ complex, served as a positive control. The $\mathrm{mAb}$ CD21 (clone BU-36), recognising an epitope on mature B lymphocytes, was used as a negative control. The cells were incubated with the primary mAbs GOH3 and $\mathrm{P} 4 \mathrm{C} 10$ at a concentration of $20-100 \mu \mathrm{g} / \mathrm{ml}$ for one hour at $4^{\circ} \mathrm{C}$. Subsequently, after washing, FBS were counterstained with the polyclonal fluorescein isothiocyanate (FITC) coupled goatantimouse antibody (Dianova-Immunotech) diluted 1:50, and placed for 45 minutes on ice. For the rat derived $\mathrm{mAb} \mathrm{GOH} 3$, a goat derived antirat $\mathrm{mAb}$ was used. After extensive washing cells were resuspended in $300 \mathrm{ml}$ of FACS medium containing $1 \mathrm{mg} / \mathrm{ml}$ propidium iodide (Sigma). Cells that had taken up propidium iodide were regarded as damaged or dying and were excluded from further analysis by gating on propidium iodide negative cells. The number of positive cells was assessed by gating on a precise upper limit of control antibody fluorescence. The interface channel for positiv- ity was set at the point where $1-5 \%$ of the control fluorescence was positive. The mean fluorescence of each experiment was calculated by subtracting mean control fluorescence values from mean fluorescence with target antibodies.

\section{STATISTICAL ANALYSIS}

Correlations between $\alpha 6 /$ laminin staining and inflammation score/treatment were calculated using the rank correlation Spearman test.

\section{Results}

INFLAMMATION SCORE, CLINICAL AND

IMMUNOSTAINING DATA

Table 1 gives the histological scores of inflammation, immunohistochemical results, and patient data. Normal synovial membranes were histologically devoid of inflammatory changes, the synovial lining layer was generally two cells in thickness. All SLC strongly expressed the $\alpha 6$ (fig 1A) and $\beta 1$ integrin subunits. In contrast with a faint pericellular laminin positivity in the synovial layer (fig 1B), subintimal arterial and venular vessels were strongly laminin positive. 
Table 2 Effect of cytokines and a synovial fluid $(S F)$ derived from a patient with $R A$ on the expression of the a6 and $\beta 1$ integrin subunits

\begin{tabular}{|c|c|c|c|c|c|c|c|c|c|}
\hline & & \multicolumn{2}{|l|}{ Medium } & \multicolumn{2}{|l|}{$I L 1 \beta$} & \multicolumn{2}{|l|}{$T N F a$} & \multicolumn{2}{|c|}{$S F$ from $R A$} \\
\hline & & \% Positive & $M F$ & $\%$ Positive & $M F$ & \% Positive & $M F$ & $\%$ Positive & $M F$ \\
\hline \multirow[t]{2}{*}{ OA-FBS 1} & $\alpha 6$ & 12.3 & 3.1 & 45 & 4.2 & 38.9 & 3.5 & 24.3 & 3 \\
\hline & $\beta 1$ & 39.3 & 13.1 & 71.7 & 15.6 & 51.5 & 15.4 & 62.2 & 20.5 \\
\hline \multirow{2}{*}{ OA-FBS 2} & $\alpha 6$ & 17.2 & 2.05 & 22.7 & 2.2 & 36.7 & 2.35 & 60.8 & 5.2 \\
\hline & $\beta 1$ & 81.7 & 19 & 95.2 & 22 & 99.6 & 25 & 96 & 23 \\
\hline \multirow[t]{2}{*}{ RA-FBS 1} & $\alpha 6$ & 65.7 & 6.1 & 8.3 & 2.3 & 4.7 & 2.1 & 44.1 & 6.1 \\
\hline & $\beta 1$ & 99.1 & 63 & 75.9 & 36.2 & 32.9 & 25 & 92.6 & 60.1 \\
\hline \multirow[t]{2}{*}{ RA-FBS 2} & $\alpha 6$ & 27.6 & 2.4 & 4.2 & 1.2 & 3.6 & 0.9 & ND & \\
\hline & $\beta 1$ & 46.7 & 25 & $\mathrm{ND}$ & & ND & & ND & \\
\hline
\end{tabular}

MF, mean fluorescence. SF from RA, medium with synovial fluid derived from a RA patient.

Table 3 Effect of anti-inflammatory drugs on the expression of the $\alpha 6$ and $\beta 1$ integrin subunits

\begin{tabular}{|c|c|c|c|c|c|c|c|c|c|}
\hline & & \multicolumn{2}{|l|}{ Medium } & \multicolumn{2}{|c|}{ Dexamethasone } & \multicolumn{2}{|c|}{ Methotrexate } & \multicolumn{2}{|c|}{ Salicylic acid } \\
\hline & & $\%$ Positive & $M F$ & $\%$ Positive & $M F$ & $\%$ Positive & $M F$ & $\%$ Positive & $M F$ \\
\hline \multirow{2}{*}{ OA-FBS 1} & $\alpha 6$ & 12.3 & 3.1 & 19.3 & 3.4 & 7 & 1.4 & 5.1 & 1.5 \\
\hline & $\beta 1$ & 39.3 & 13.1 & 16 & 10.4 & 27.4 & 11.2 & 43.4 & 13.6 \\
\hline \multirow{2}{*}{ OA-FBS 3} & $\alpha 6$ & 12.5 & 3.2 & 6.3 & 2.3 & 5.3 & 1.7 & 7.6 & 2.5 \\
\hline & $\beta 1$ & 86 & 21 & 51 & 24 & 34.6 & 13 & 60 & 25 \\
\hline \multirow[t]{2}{*}{ RA-FBS 3} & $\alpha 6$ & 2.8 & 5.5 & 24.6 & 8.4 & 22.4 & 6.3 & 6.4 & 5.8 \\
\hline & $\beta 1$ & 26.8 & 33 & 84.6 & 49 & 97.3 & 76 & 22.1 & 23.2 \\
\hline \multirow[t]{2}{*}{ RA-FBS 4} & $\alpha 6$ & 17.4 & 4.05 & 25.5 & 5.5 & 45.2 & 6 & 21.2 & 4.5 \\
\hline & $\beta 1$ & 55.3 & 11.8 & 61 & 20 & 69.2 & 13 & 57.2 & 16 \\
\hline
\end{tabular}

With regard to the expression of the $\beta 1$ integrin subunit, all or the majority of SLC in 12 of 15 OA samples and in 18 of 19 RA samples were strongly positive. By contrast, $\alpha 6$ and laminin staining in SLC of OA and RA samples was rather heterogeneous.

OA samples presented a higher histological score of inflammation (mean (SD), $6.2(1.9)$ ). The histological score correlated with the thickness of the synovial intima $(p=0.019)$. Within this group 6 of 15 presented an inflammation scoring value between 4 and 5 (samples $3,7,8,10,13$, and 16), whereas the other OA synovia samples had a higher score of inflammation $(\geqslant 6)$.

In 7 of 15 samples (samples 3, 5, 6, 8, 9, 14, and 16) the majority of SLC showed $\alpha 6$ and laminin staining. However, as with normal synovia, laminin staining was weaker in comparison with $\alpha 6$. In these OA synovia samples SLC were usually flat and distributed in a low number of cell layers. In 4 of $15 \mathrm{OA}$ samples (samples $7,10,11$, and 15) a faint laminin positivity was found in a low number of SLC, whereas $\alpha 6$ was strongly expressed and distributed in a high number of SLC (fig 1C, D). In 4 of 15 OA samples (samples 4,12,13, and 17) only a subset of SLC (1-25\%) was $\alpha 6$ positive and showed laminin staining. Three of these four OA samples presented a higher degree of inflammation $(\geqslant 7)$ in comparison with the other OA samples. No correlation was found between the degree of inflammation, $\alpha 6$ and laminin staining, and the joint site of the corresponding synovia samples. Inflammation scores of the detected OA synovia samples did not correlate with the laboratory parameter erythrocyte sedimentation rate (ESR).

RA samples presented a high grade of inflammation (mean (SD), 10.4 (3.2)). The degree of inflammation correlated statistically $(p=0.010)$ with the synovial lining thickness, but no correlation was found between the degree of inflammation and laboratory tests (ESR, rheumatoid factor (RF)).

In 3 of 19 RA synovia samples $\alpha 6$ and laminin were distributed in a large number of SLC (samples 21, 22, and 36). In 12 of $19 \mathrm{RA}$ synovia samples laminin staining was restricted to a small number of SLC (samples $18,19,23,26,27,29,30,31$, and 32 ), or was negative (samples 20,33, and 34) in comparison with the number of the $\alpha 6$ positive. SLC had a plump cell shape with strong $\alpha 6$ positivity confined to the surface cell membrane. In 3 of 19 RA samples (samples 25, 28, and 35) $\alpha 6$ and laminin were distributed only in one subset of SLC, whereas in 1 of 19 RA samples (sample 24) SLC lacked these antigens (fig $1 \mathrm{E}, \mathrm{F}) . \alpha 6$ and laminin staining inversely correlated with the inflammation score $(\mathrm{p}=$ 0.004 , and 0.006 , respectively) and with the synoviocyte hyperplasia $(\mathrm{p}=0.034$, and 0.008 , respectively). $\alpha 6$ and laminin staining decreased in RA samples with a high grade of inflammation and with hypertrophic lining layers. However, the decrease in laminin staining was stronger than in $\alpha 6$ staining. No correlations were found between $\alpha 6$, laminin staining and treatment or joint site of origin of the synovia samples.

EXPRESSION OF THE A6 AND B1 SUBUNITS ON CYTOKINE AND DRUG TREATED FBS

To investigate whether cytokines and drugs influence the expression of $\alpha 6$ and $\beta 1$, long term cultures derived from OA and RA synovia were kept in the presence of cytokines (IL1 $\beta$ and $\mathrm{TNF} \alpha$ ) and of drugs (dexamethasone, methotrexate, and salicylic acid), and were analysed for the expression of $\alpha 6$ and $\beta 1$ by FACS analysis (tables 2 and 3). Having treated OA-FBS with IL $1 \beta$ and TNF $\alpha$ we observed an increase in $\alpha 6$ and $\beta 1$ expression on the two detected FBS cultures of OA. The most prominent effect on the expression of $\alpha 6$ was 

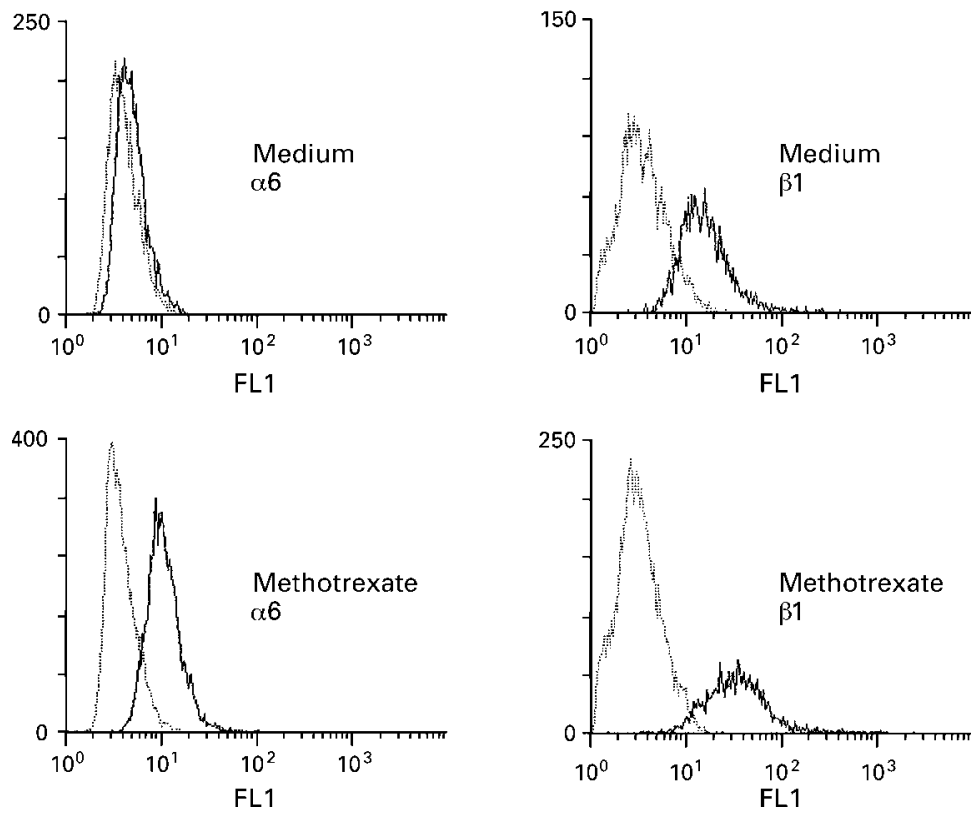

Figure 2 Histograms of flow cytometric analysis for the $\alpha 6$ and $\beta 1$ integrin subunits on untreated (upper panels) and with methotrexate treated (lower panels) FBS from RA. The fluorescence obtained with isotypic negative control antibodies is plotted as a plain line, the results with anti-a6 as a bold line.

induced by IL1 $\beta$ in OA- FBS 1 and by TNF $\alpha$ in OA-FBS 2. The most relevant up regulating effect on the expression of $\beta 1$ was obtained by IL1 $\beta$ in OA-FBS 1 , whereas in OA-FBS 2 , IL1 $\beta$ and TNF $\alpha$ had a similar effect.

The effect of cytokines was different in RA-FBS. The expression of $\alpha 6$ was markedly reduced by treament with IL $1 \beta$ and TNF $\alpha$ in RA-FBS 1 and RA-FBS 2. In RA-FBS 1 the maximal reduction on $\beta 1$ expression was obtained by treatment with TNF $\alpha$.

To reproduce in our model in vitro the effect of factors that play a part in the inflammatory process in vivo, OA-FBS and RA-FBS were cultured in the presence of the supernatant of a synovial fluid obtained from an arthritic joint of a patient with RA. Treatment of OA-FBS with this synovial fluid up regulated the $\alpha 6$ and $\beta 1$ expression in both FBS cultures, whereas the addition of this synovial fluid to RA-FBS decreased the expression of $\alpha 6$ and only minimally decreased $\beta 1$ in one of the RA-FBS cultures.

Drug treatment of OA-FBS resulted in a down regulating effect on the expression of $\alpha 6$. This effect was observed in particular using methotrexate and salicylic acid in the two studied OA-FBS cultures and using dexamethasone in OA-FBS 3 (table 3). Treatment of OA-FBS with dexamethasone and methotrexate resulted in a down regulating effect on the expression of the $\beta 1$ on OA-FBS 1 and 3. This effect was also observed upon treating OA-FBS 3 with salicylic acid.

In contrast with these findings treatment of RA-FBS with the same drugs up regulated the expression of the $\alpha 6$ and $\beta 1$ integrin subunits (table 3 ). The most prominent up regulating effect on $\alpha 6$ and $\beta 1$ of RA-FBS was observed with dexamethasone and salicylic acid and, more intensely, with methotrexate (fig 2).

\section{Discussion}

We have shown in accordance with other authors $^{1314}$ a faint laminin positivity in the synovial layer of normal synovia. As FBS have been shown to synthesise laminin ${ }^{2}$ the intense laminin staining found in the synovia of OA and RA with mild inflammation may be because of the proliferation of the synovial cell layers. In contrast with this situation, in the severe inflammation of OA and RA there was hyperplasia of the SLC and we observed a decrease in the amounts of laminin, which was dispersed in the synovial lining layer and limited to a restricted number of SLC. These findings suggest that the decrease in laminin is associated with the change of intensity of inflammation. However, laminin was absent only in synovia samples of RA with a high grade of inflammation. The presence of laminin in the synovia from chronic synovitis of different aetiology can be related to a different synthesis of laminin by fibroblast-like synovial cells. Alternatively, the decrease in laminin protein may be because of the remodelling process of the extracellular matrix components by metalloproteinases. ${ }^{15-17}$

Cell interaction with the extracellular matrix components is mediated by integrins. ${ }^{3}$ Given the important role of the matrix in the regulation of cellular functions, changes in the extracellular matrix protein laminin can be responsible for a differential integrin pattern. ${ }^{18-20}$ The best known integrin receptors for laminin are $\alpha 6 \beta 1$ and $\alpha 6 \beta 4$. $^{321}$ As SLC have been shown to lack $\beta 4, \alpha 6$ most probably associates with $\beta 1$ to form receptors for laminin. ${ }^{14}$ Supporting the in situ data, we showed in a recent report, ${ }^{22}$ that binding of cultured fibroblast-like synovial cells to laminin is mediated by the $\alpha 6$ and $\beta 1$ integrins but not by $\beta 4$ integrins. The consistent expression of the $\beta 1$ subunit in all synovia samples studied corresponds to data from the literature. ${ }^{11}{ }^{1423-25}$ One explanation for this finding is that $\beta 1$ associates with several $\alpha$ integrin subunits to form receptors for different extracellular matrix proteins, as demonstrated in blocking experiments showing that antibodies to $\beta 1$ integrins inhibit binding to collagen type IV, fibronectin and laminin on FBS in vitro. ${ }^{22}$

In normal synovia and in $\mathrm{OA}$ synovia samples with a low grade of inflammation the distribution of the $\alpha 6$ integrin subunit paralleled laminin staining. The expression of $\alpha 6$ is not associated with a similar pattern of laminin staining in OA synovia samples with a higher grade of inflammation and in the majority of the RA synovia samples. Laminin was distributed in a pericellular manner in a limited number of SLC, whereas $\alpha 6$ was surface expressed in a high number of cells. This may suggest that SLC, in response to a decrease in laminin, react with an up regulation of the $\alpha 6$ molecules trying to provide a major surface contact with the ligand laminin. In synovia samples of RA with severe inflammation we observed, parallel to the loss of laminin, a decrease in $\alpha 6$ staining. A possible explanation could be that with the complete lack of the ligand, SLC down regulate the production of the 
$\alpha 6$ molecules. We and others have shown ${ }^{14}$ that a6 staining inversely correlates with the synovial lining thickness. It is still open to question whether changes in $\alpha 6$ and in laminin staining are typical for RA.

To substantiate further our in situ findings, in vitro studies on FBS cultures derived from $\mathrm{OA}$ and RA synovia samples were carried out. In particular, we studied the effect of cytokines and of drugs, on the expression of the $\alpha 6$ and $\beta 1$ integrin subunits. Even if there were differences in the baseline expression of $\alpha 6$ and $\beta 1$ on the FBS cultures studied, the effect of cytokines and drugs on the expression of these integrins was specific for RA-FBS and OAFBS and regardless of the original level of the integrin subunits. In particular IL1 $\beta$ and $\mathrm{TNF} \alpha$ increased the expression of $\alpha 6$ and $\beta 1$ on OA-FBS, whereas these cytokines decreased $\alpha 6$ and $\beta 1$ expression on RA-FBS. An analogous effect was seen upon treatment of the FBS with a synovial fluid obtained from an arthritic joint of a patient with RA. IL1 $\beta$ and $\mathrm{TNF} \alpha$ are involved in inflammation induced tissue damage and joint destruction. ${ }^{26}$ Our findings suggest that these cytokines regulate the $\alpha 6$ expression in vivo. Our data in vitro on the cytokine stimulated RA-FBS can explain the decrease of $\alpha 6$ in SLC of RA synovia with a high grade of inflammation. In contrast with these findings we showed that antirheumatic drugs such as salicylic acid and dexamethasone and in particular methotrexate had an up regulating effect on the expression of $\alpha 6$ and $\beta 1$ on RA-FBS raising the possibility that an increase in $\alpha 6$ and $\beta 1$ expression can provide a protective effect on the cells. Further studies are necessary to investigate whether these effectors regulate the synthesis of laminin by cultured FBS.

Taken together, the loss of the laminin receptor integrin subunit $\alpha 6$ and of its ligand in the rheumatoid synovia represents a change associated with inflammation. As laminin is a matrix component involved in the structural organisation of the synovia, the loss of laminin may contribute to the damage of the synovia in chronic synovitis. Furthermore, we have shown that IL1 $\beta$ and TNF $\beta$ decrease the expression of $\alpha 6$ on RA-FBS, suggesting that these cytokines may modulate the $\alpha 6$ expression on SLC in the rheumatoid process in vivo.

We would like to thank U Fabian and E Michalek for technica assistance.

1 Castor CW. The microscopic structure of normal human

2 Revell PA, Al-Saffar N, Fish S, Osei D. Extracellular matrix

of the synovial intimal cell layer. Ann Rheum Dis 1995;54: of the syn.
3 Hynes RO. Integrins: versatility, modulation, and signaling in cell adhesion. Cell 1992;69:11-25.

4 Ruoslahti E, Noble NA, Kagami S, Border WA. Integrins. Kidney Int 1994;45:17-22.

5 Otey CA, Pavalko FM, Burridge K. An interaction between $\alpha$-actinin and the $\beta 1$ integrin subunit in vitro. J Cell Biol 1990;111:721-9.

6 Cutolo M, Picasso M, Ponassi M, Zhen Sun M, Balza E. Tenascin and fibronectin distribution in human normal and pathological synovium. J Rheumatol 1992;19:1439and.

7 McCachren SS, Lightner VA. Expression of human tenascin in synovitis and its regulation by interleukin-1. Arthritis Rheum 1992;35:1185-6.

8 Altman R, Asch E, Bloch D, Bole G, Borenstein D, Brandt $\mathrm{K}$, et al. Development of criteria for the classification and reporting of osteoarthritis: classification of osteoarthritis of the knee. Arthritis Rheum 1988;29:1039-49.

9 Arnett FC, Edworthy SM, Bloch DA, McShane DJ, Fries $\mathrm{JF}$, Cooper NS, et al. The American Rheumatism Association 1987 revised criteria for die classification of rheumatoid arthritis. Arthritis Rheum 1988;31:315-24.

10 Abrahamsen TG, Froland SS, Natvig JB, Pahle J. Elution and characterization of lymphocytes from rheumatoid inflammatory tissue. Scand J Immunol 1975;4:822-30.

11 Rinaldi N, Barth T, Henne C, Mechtersheimer G, Möller P. Synoviocytes in chronic synovitis in situ and cytokine stimulated synovial cells in vitro neo-express $\alpha 1, \alpha 3$ and $\alpha 5$ chains of $\beta 1$ integrins. Virchows Arch 1994;425:171-80.

12 Rooney M, Condell D, Quinlan W, Daly L, Whelan A, Feighery C, et al. Analysis of the histologic variation of synovitis in rheumatoid arthritis. Arthritis Rheum 1988;31: $956-63$.

13 Pollock LE, Lalor P, Revell PA. Type IV collagen and aminin in the synovial intimal layer: an immunohistochemical study. Rheumatol Int 1990;9:277-80.

14 Nikkari L, Aho A, Yli-Jama, T, Larjava H, Jalkanen M, Heino J. Expression of integrin family of cell adhesion receptors in rheumatoid synovium. Alpha 6 integrin subunit in normal and hyperplastic synovial lining cell layer. Am nit in normal and hyperplastic

15 Woessner JF. Matrix metalloproteinases and their inhibitors in connective tissue remodeling. FASEB J 1991;5:214554

16 Murphy G, Cockett MI, Ward RV, Docherty AJ. Matrix metalloproteinase degradation of elastin, type IV collagen and proteoglycan: A quantitative comparison of the activities of $95 \mathrm{kDa}$ and $72 \mathrm{kDa}$ gelatinases, stromelysins -1 and -2 and punctuated metalloproteinase (PUMP). Biochem J 1991;277:277-9.

17 Imai K, Yokohama Y, Nakanishi I, Onochi E, Fujii Y, Nakai $\mathrm{N}$, et al. Matrix metalloproteinase 7 (matrilysin) from human rectal carcinoma cells. Activation of the precursor, human rectal carcinoma cells. Activation of the precursor,
interaction with other matrix metalloproteinases and interaction with other matrix metalloproteinases
enzymic properties. J Biol Chem 1995;270:6691-7.

18 Juliano RL, Haskill S. Signal transduction from the extracellular matrix. J Cell Biol 1993;120:577-85.

19 Lévesque JP, Hatzfeld A, Hatzfeld J. Mitogenic properties of extracellular matrix proteins. Immunmol Today 1991;12: 258-62.

20 Grinnell F, Toda KI, Takashima A. Activation of keratinocyte fibronectin receptor function during cutaneous wound yte fibronectin receptor function during

21 Sonnenberg A, Linders CJT, Daams JH, Kennel SJ. The $\alpha 6 \beta 1$ (VLA-6) and the $\alpha 6 \beta 4$ protein complexes: tissue distribution and biochemical properties. J Cell Sci 1990;96: 207-16.

22 Rinaldi N, Schwarz-Eywill M, Weis D, Leppelmann-Jansen P, Lukoschek M, Keilholz U, Barth TFE. Increased expression of integrins on fibroblast-like synoviocytes from rheumatoid arthritis in vitro correlates with enhanced binding to extracellular matrix proteins. Ann Rheum Dis 1997;56: 45-51.

23 Demaziere A, Athanasou NA. Adhesion receptors of intimal and subintimal cells of the normal synovial membrane. J Pathol 1992;168:209-15.

24 Johnson BA, Haines GK, Harlow LA, Koch. AE. Adhesion molecule expression in human synovial tissue. Arthritis Rheum 1993;36:137-46.

25 El-Gabalawy H, Wilkins J. $\beta 1$ (CD29) integrin expression in rheumatoid synovial membranes: an immunohistologic study of distribution patterns. J Rheumatol 1993;20:231-7.

26 Lipsky PE, Davis LS, Cush JJ, Oppenheimer-Marks N. The role of cytokines in the pathogenesis of rheumatoid arthritis. Springer Semin Immunopathol 1989;11:123-62. 